\title{
TLS POINT CLOUD REGISTRATION FOR DETECTING CHANGE IN INDIVIDUAL ROCKS OF A MOUNTAIN RIVER BED
}

\author{
A. Walicka ${ }^{1 *}$, N. Pfeifer ${ }^{2}$, G. Jóźków ${ }^{1}$, A. Borkowski ${ }^{1}$ \\ ${ }^{1}$ Institute of Geodesy and Geoinformatics, Wrocław University of Environmental and Life Sciences, Wrocław, Poland \\ (agata.walicka, grzegorz.jozkow, andrzej.borkowski)@upwr.edu.pl \\ ${ }^{2}$ Department of Geodesy and Geoinformation, TU Wien, Vienna, Austria \\ (norbert.pfeifer@geo.tuwien.ac.at)
}

Commission II, WG II/3

KEY WORDS: Terrestrial laser scanning, Iterative Closest Point algorithm, ICP, point cloud registration, sediment transport monitoring

\begin{abstract}
:
Remote sensing techniques are an important tool in fluvial transport monitoring, since they allow for effective evaluation of the volume of transported material. Nevertheless, there is no methodology for automatic calculation of movement parameters of individual rocks. These parameters can be determined by point cloud registration. Hence, the goal of this study is to develop a robust algorithm for terrestrial laser scanning point cloud registration. The registration is based on Iterative Closest Point algorithm, which requires well established initial parameters of transformation. Thus, we propose to calculate the initial parameters based on key points representing the maximum of Gaussian curvature. For each key point the set of geometrical features is calculated. The key points are then matched between two point clouds as a nearest neighbor in feature domain. Different combinations of neighborhood sizes, feature subsets, metrics and number of nearest neighbors were tested to obtain the highest ratio between properly and improperly matched key points. Finally, RANSAC algorithm was used to calculate the initial transformation parameters between the point clouds and the ICP algorithm was used for calculation of final transformation parameters. The investigations carried out on sample point clouds representing rocks enabled the adjustment of parameters of the algorithm and showed that the Gaussian curvature can be used as a 3-dimentional key point detector for such objects. The proposed algorithm enabled to register point clouds with the mean distance between point clouds equal to $3 \mathrm{~mm}$.
\end{abstract}

\section{INTRODUCTION}

One of the tasks undertaken as a part of hydrological and geomorphological research is fluvial transport monitoring. The knowledge of the movement of different rock fractions in the river bed enables to predict future changes in surface relief formation, and to determine the river bed parameters.

Because of its complicated nature, fluvial transport investigation requires high quality sedimentary measurements. Traditionally, this measurements are performed using sediment traps (e.g. Garcia et al., 2000; Bergman et al., 2007) or tracers (e. g. Olinde \& Johnson, 2015; Ancey \& Heyman, 2014). Nowadays, to support these methods and to avoid toilsome field work, various remote sensing techniques are being introduced to this research field.

Many researchers (e.g. Picco et al., 2013; Kuo et al., 2015; Barnhart et al., 2013; Bertin et al., 2015) demonstrated that the remote sensing techniques allow for an effective and fast estimation of the volume of dislocated rock material. However, determining movement parameters of individual rocks is still a challenging task, because a rock can be not only displaced but also rotated in 3D space causing that different part of stone surface is scanned during different data acquisition campaigns. The research conducted by Jóźków et al. (2016) and Lotsari et al. (2015) showed that the horizontal movement of individual rocks can be investigated by means of laser scanning point cloud data analysis, but in both cases the detection of individual stone movements was performed manually. Therefore, an algorithm for an automatic calculation of individual rock movement parameters is needed.

The overall goal of this research is to automatically determine the movement of individual rocks, based on multi-temporal point clouds. The specific goal of this study is to develop a robust algorithm for two-epoch point cloud registration. The approach is built on three assumptions. Firstly, two point clouds were acquired during different epochs. As a result, the shape of objects may be slightly changed between the scans. Secondly, objects represented by the point clouds have oblong, rounded shape. Finally, one of point clouds represents a wider scene than the other. An example of such case is shown in Figure 1.

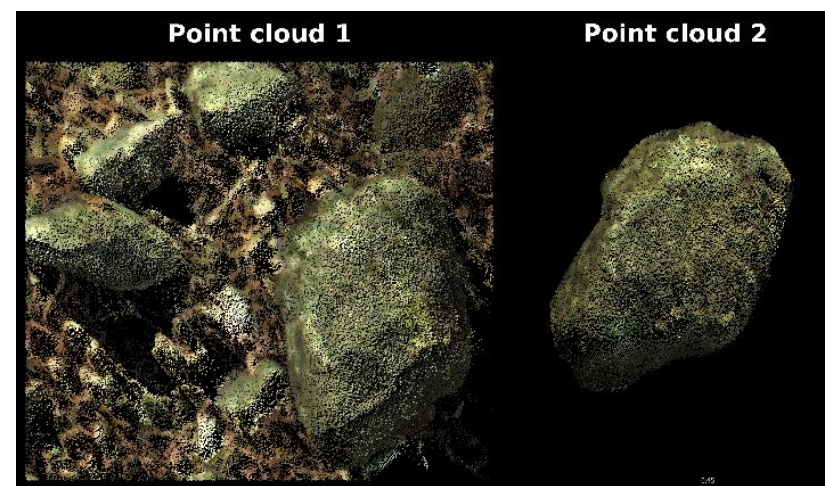

Figure 1. Example of terrestrial laser scanning point clouds under registration; reference point cloud - point cloud 1; aligned point cloud - point cloud 2.

\footnotetext{
* Corresponding author
} 


\section{TEST SITE AND DATA ACQUISITION}

The study area is located in south-western Poland in Giant Mountains, the highest part of Sudeten. Łomniczka river was chosen as the research object based on its two features: the river bed contains a lot of large-size rocks and during the autumn the water level is low, what enables measurements of lower parts of stones.

The measurements were carried out annually in years 2011-2017. The data was collected by means of terrestrial laser scanner - Leica ScanStation C10 and Leica ScanStation P20. The measurements were performed from two scanner positions. The resulting point clouds were co-registered and georeferenced based on three evenly distributed targets, which coordinates were determined with centimeter-level accuracy.

The measurements resulted in creation of 7 point clouds - one for each year. For the purposes of this research, the selected parts of point clouds acquired in years 2011 and 2013 were used. The density of the point clouds is approximately 119000 points $/ \mathrm{m}^{2}$.

\section{METHODOLOGY}

\subsection{ICP algorithm}

To register point clouds, the Iterative Closest Point (ICP) algorithm was chosen. The ICP algorithm is a method to coregister two or more point clouds by iterative minimization of the distance between corresponding points from two point clouds. The algorithm finds corresponding points in point clouds based on closest point assumption. The algorithm can be divided into five steps (Glira et al., 2015):

1) Selecting a subset of points lying in the overlap area of two point clouds.

2) Finding correspondences of selected points in the second point cloud.

3) Rejecting false correspondences.

4) Calculating the transformation parameters between the point clouds.

5) Transformation of the point cloud 2 based on calculated parameters.

To register the point clouds, the ICP algorithm implemented in OPALS (Glira et al., 2015) software was chosen. However, the ICP algorithm requires initial parameters of transformation, especially when the significant rotation occurs. To solve this problem, we suggest to find and match key points in the point clouds.

\subsection{Key points detection}

The identification of key points was performed based only on geometrical information. Firstly, the data was smoothed to avoid false key point detection. To do this, the Moving Least Squares algorithm implemented in Point Cloud Library was used (Rusu $\&$ Cousins, 2011). Then the Gaussian curvature was calculated based on local neighborhood. The choice of Gaussian curvature as a geometrical feature describing key points was caused by the specific, oblong shape of rocks. Because of this shape, proper identification of key points by standard algorithms, which base only on geometry information (e.g. Intrinsic Shape Signatures Zhong Y., 2009) was impossible due to the lack of detected key points.

In the next step, the local maxima of Gaussian curvature were found using the algorithm shown in Figure 2. At the beginning, the point cloud is loaded. Each point has the following attributes: $x$, $y$ planar coordinates, $h$ - height, and $c$ - Gaussian curvature. All points in the point cloud are marked as 'not visited'. Next, the KDTree is built for fast neighboring points selection. Then the first point $k$ marked as 'not visited' is selected. After that, for this point, a spherical neighborhood of the radius $r$ is investigated. Then, the maximum value of Gaussian curvature in this neighborhood is found. If the maximum value belongs to the point $k$, all points in the neighborhood are marked as 'visited', point $k$ is marked as local maximum, and the next point marked as 'not visited' is selected as the new point $k$. Otherwise, all points in the neighborhood having Gaussian curvature lower than points $k$ curvature are marked as 'visited' and the next point marked as 'not visited' is selected as the new point $k$. After that, all steps are repeated until all points in the point cloud are marked as 'visited'. As a result, a set of points representing local maxima and consequently key points is constructed.

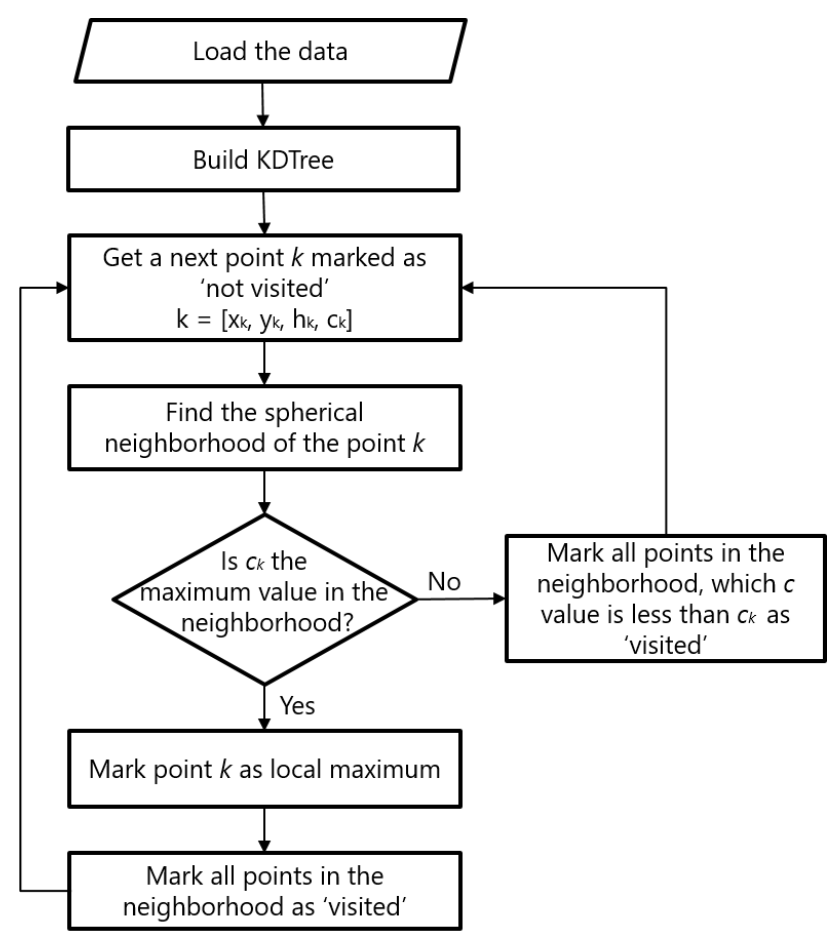

Figure 2. Local maxima detection algorithm

\subsection{Key points matching}

The key point matching was based only on geometrical information. For each key point the set of local descriptors was calculated based on spherical neighborhood of these points. The following features were calculated:
- Sphericity
$S_{\lambda}=\frac{\lambda_{3}}{\lambda_{1}}$
- Linearity
$L_{\lambda}=\frac{\lambda_{1}-\lambda_{2}}{\lambda_{1}}$
- Planarity
$P_{\lambda}=\frac{\lambda_{2}-\lambda_{3}}{\lambda_{1}}$
- $\quad$ Anisotropy
$A_{\lambda}=\frac{\lambda_{1}-\lambda_{3}}{\lambda_{3}}$
$O_{\lambda}=\sqrt[3]{\prod_{i=1}^{3} \lambda_{i}}$
- Omnivariance 


$$
\begin{aligned}
& \text { - Eigentropy } \quad E_{\lambda}=-\sum_{i=1}^{3} \lambda_{i} \ln \left(\lambda_{i}\right) \\
& \text { - Local surface variance } \quad C_{\lambda}=\frac{\lambda_{3}}{\sum_{i=1}^{3} \lambda_{i}} \\
& \text { - Sum of eigenvalues } \quad \Sigma_{\lambda}=\sum_{i=1}^{3} \lambda_{i}
\end{aligned}
$$

- $\quad$ Number of points in the local neighborhood

- Gaussian curvature

Where $\lambda_{i}, i=1,2,3$ are singular values of the covariance matrix calculated for coordinates of neighboring points.

Based on calculated local descriptors, matching key points were selected for the point cloud representing a single stone. The matching points were indicated as $n$ nearest neighbors in the feature domain. The nearest neighbors were selected based on one of three metrics: Manhattan (Equation 9), Euclidean (Equation 10), and Maximum (Equation 11). Different combinations of neighborhood sizes, feature subsets, metrics, and number of nearest neighbors were tested on a rock sample to obtain the highest rate (Equation 12) between the number of properly and improperly identified key point pairs.

$$
\begin{aligned}
& d_{\text {Manhattan }}=\left|x_{1}-x_{2}\right|+\left|y_{1}-y_{2}\right|+\left|z_{1}-z_{2}\right| \\
& d_{\text {Euclidean }}=\sqrt{\left(x_{1}-x_{2}\right)^{2}+\left(y_{1}-y_{2}\right)^{2}+\left(z_{1}-z_{2}\right)^{2}} \\
& d_{\text {Max }}=\max \left(\left|x_{1}-x_{2}\right|,\left|y_{1}-y_{2}\right|,\left|z-z_{2}\right|\right)
\end{aligned}
$$

where:

$d_{\text {Manhattan }}$ - distance between two 3D-points in Manhattan metric

$d_{\text {Euclidean }}$ - distance between two 3D-points in Euclidean metric $d_{\text {Max }}$ - distance between two 3D-points in Maximum metric

$x_{i}, y_{i}, z_{i}$ - coordinates of point belonging to $i$-th point cloud

$$
R=\frac{\text { number of properly identified key point pairs }}{\text { number of improperly identified key point pairs }}
$$

\subsection{Initial parameters calculation}

As a result of the further processing, the list of best matching point pairs between the point clouds has been created. Wherein, to each key point in the point cloud representing stone, the $n$ points in the larger point cloud are assigned. As a result, both correct and incorrect assignments have been included in the created list. Because of that, the Random Sample Consensus (RANSAC) algorithm was used for robust calculation of initial parameters for the ICP algorithm.

RANSAC algorithm is an iteration-based method designed for robust fitting model to experimental data. In each step of the processing, RANSAC algorithm randomly selects required number of points from each dataset and, based on these points, calculates the parameters of the model. After that, number of points compatible with the model is counted. The algorithm terminates when one of the following conditions is met:

1) When the specified number of points is found compatible, the transformation parameters calculated in this iteration are chosen as final model parameters.

2) When the specified number of iterations is finished, the transformation parameters with the biggest number of points compatible with the model are chosen as the final model parameters.

Therefore, the RANSAC algorithm needs three parameters to be specified: the number of subsets to try, the number of compatible points that imply that the correct model parameters have been found, and the threshold to decide if the point is compatible with the model (Fischler and Bolles, 1981).

For the purposes of this research, the possible combinations of points in both point clouds were limited to the matching points found in previous step. As a result, in each iteration of RANSAC algorithm 3 pairs of matching points were selected. Then, the rigid transformation parameters were calculated based on these pairs. Next, the key points detected in aligned (point cloud 2) point cloud were transformed using calculated parameters and the distance between matching key points was calculated. The initial parameters with the biggest number of compatible points were chosen.

During the experiments, different combinations of threshold values, and number of iterations were tested. The number of compatible points that imply that the correct model parameters were found has been defined as the number of key points in point cloud representing stone.

\section{RESULTS}

\subsection{Key points detection}

Before Gaussian curvature calculation, each point cloud was smoothed using Moving Least Squares algorithm implemented in Point Cloud Library with the following parameters:

- $\quad$ search radius $-5 \mathrm{~cm}$

- $\quad$ polynomial order -2 .

The results of smoothing performed on example point cloud are shown in Figure 3.

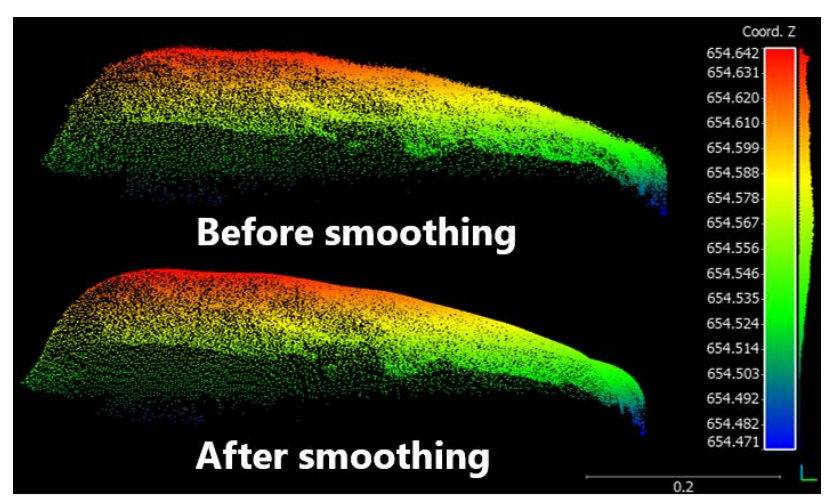

Figure 3. Smoothing results

Then the Gaussian curvature was calculated and local maxima were detected. Due to the specificity of the algorithm used for local maxima detection, the size of the neighborhood is the most important factor for the quality and the number of key points. Therefore, different sizes of the neighborhood were tested on three randomly chosen rock samples of different sizes. Five different diameters of the neighborhood were examined - from 2 to $6 \mathrm{~cm}$ every $1 \mathrm{~cm}$. The results were visually interpreted and the neighborhood size of $3 \mathrm{~cm}$ was chosen as a compromise between the processing time, number of key points, and key point location with respect to Gaussian curvature value. The results of key point detection in point clouds acquired in years 2011 and 2013 are shown in Figure 4. 


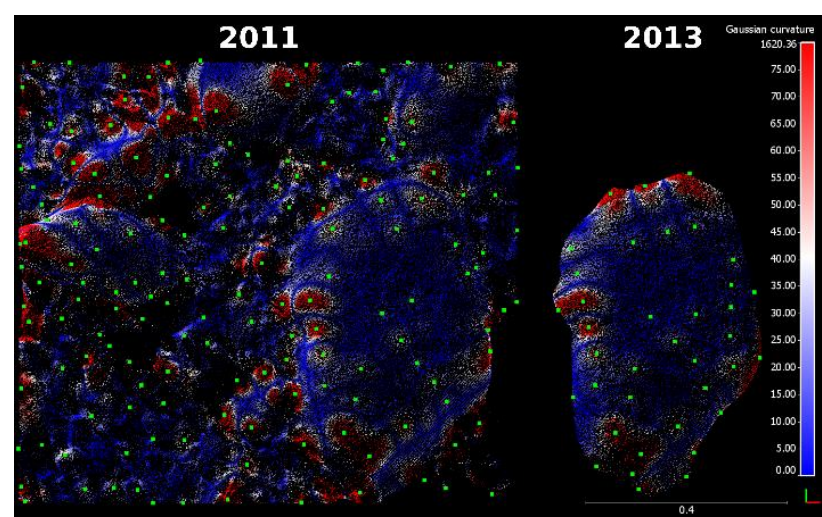

Figure 4. Key point detection results for the neighborhood size of $3 \mathrm{~cm}$. Blue, white, red - Gaussian curvature, green - key points

\subsection{Key point matching}

Before feature calculation and key point matching five parameters have to be defined:

- radius for feature calculation,

- feature vector length,

- feature combination,

- metric used for finding nearest neighbors,

- $\quad$ number of nearest neighbors.

To obtain optimal result, different combinations of parameters were tested. The experiments included:

- 4 radius sizes $-0.03 \mathrm{~m}, 0.05 \mathrm{~m}, 0.1 \mathrm{~m}, 0.2 \mathrm{~m}, 0.3 \mathrm{~m}$;

- 9 lengths of feature vector;

- 511 different combinations of features;

- 3 metrics - Manhattan, Euclidean, and maximum;

- 10 numbers of nearest neighbors - from 1 to 10 .

The achieved results are shown in the Figures 5-7. The points indicate test cases. The color is marked with the value of rate between the number of properly and improperly identified key point pairs (Equation 9). The maximum value of the $R$ rate (0.42) was achieved for one of the test cases. As a result the following combination of parameters was chosen:

- $\quad$ radius size $-0.1 \mathrm{~m}$;

- $\quad$ length of feature vector -6 ;

- combination of features - Gaussian curvature, sphericity, linearity, anisotropy, local surface variance, sum of eigenvalues;

- metric - Manhattan;

- number of nearest neighbors -1 .

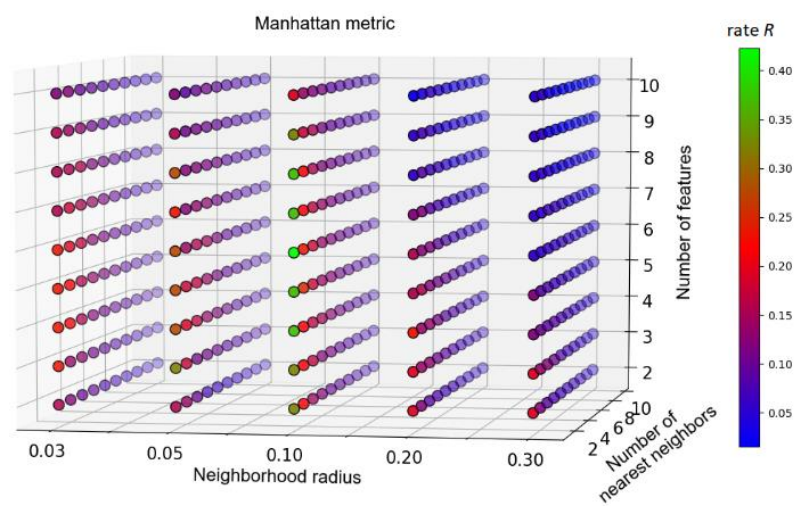

Figure 5. Results of the experiments for Manhattan metric

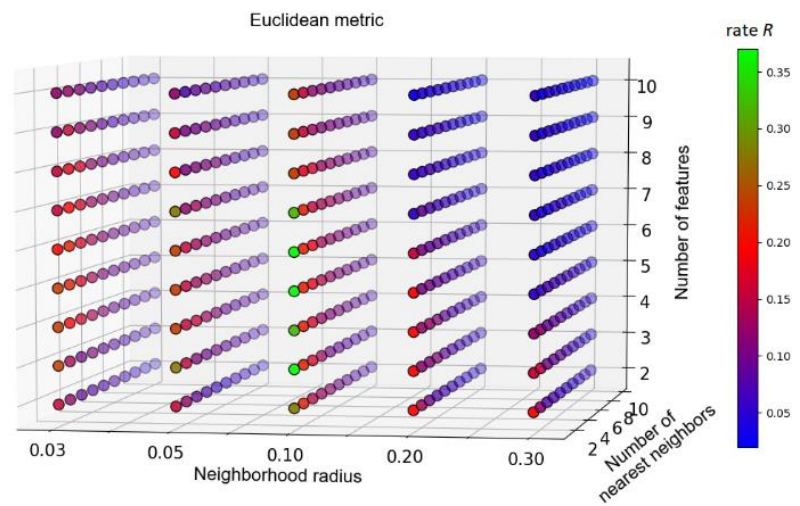

Figure 6. Results of the experiments for Euclidean metric

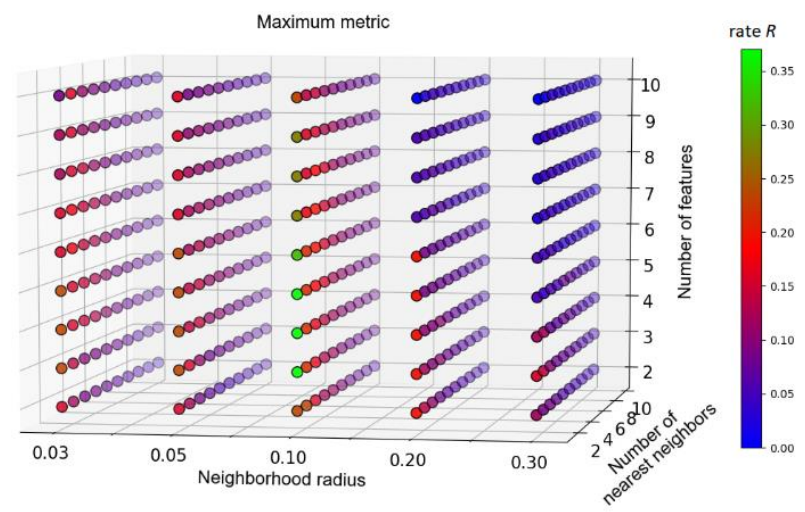

Figure 7. Results of the experiments for maximum metric

\subsection{Registration}

The registration was divided into two steps. Firstly, the RANSAC algorithm based on matched key point pairs was used to calculate the initial transformation parameters. In this step, the experiments were focused on choosing the best parameters for RANSAC algorithm. The threshold values of $3 \mathrm{~cm}, 5 \mathrm{~cm}, 10 \mathrm{~cm}, 15 \mathrm{~cm}$, and $20 \mathrm{~cm}$ and iteration numbers of 10, 50, 100, 500, and 1000. Each parameter combination was tested 100 times. The results were evaluated based on value of mean distance between point clouds transformed manually and automatically. The results of the experiments are shown in the Figure 8. The smallest error was achieved for $5 \mathrm{~cm}$ threshold value and 1000 iterations. Therefore, these parameters were chosen as the optimal ones. Secondly, the ICP algorithm implemented in OPALS software was applied to calculate final transformation parameters. The proposed algorithm enabled to register the point clouds and to achieve $0.003 \mathrm{~m}$ mean distance between point clouds.

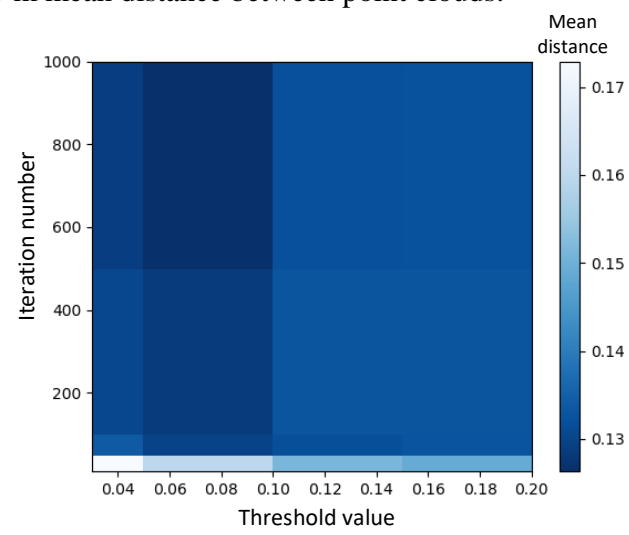

Figure 8 Results of the experiments with RANSAC parameters 
After the parameters adjustment, the algorithm was launched on two validation sets representing different rocks located in the Łomniczka river bed. The proposed algorithm enabled to register the point clouds and to achieve less than 0.003 m mean distance between point clouds. An example result of the proposed algorithm is shown in Figure 9 -11.

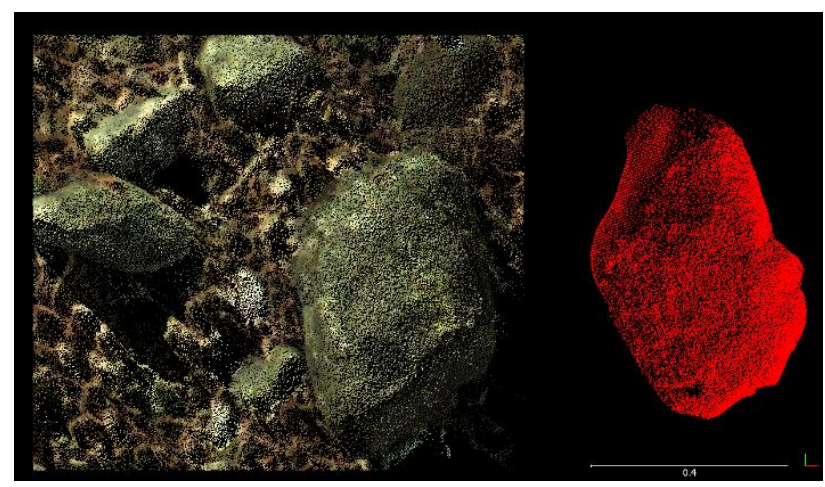

Figure 9 Initial position of point clouds; red - aligned point cloud

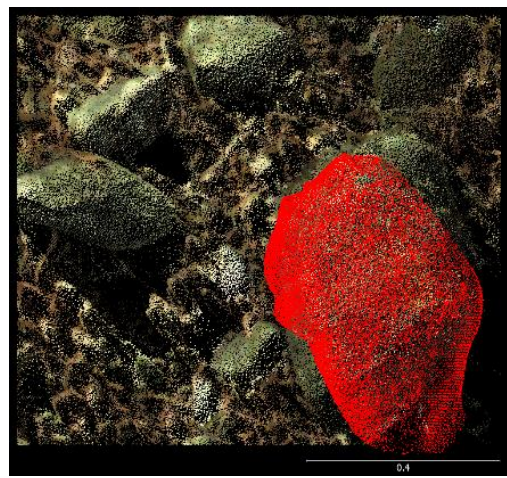

Figure 10 Position of point clouds after initial transformation, red - aligned point cloud

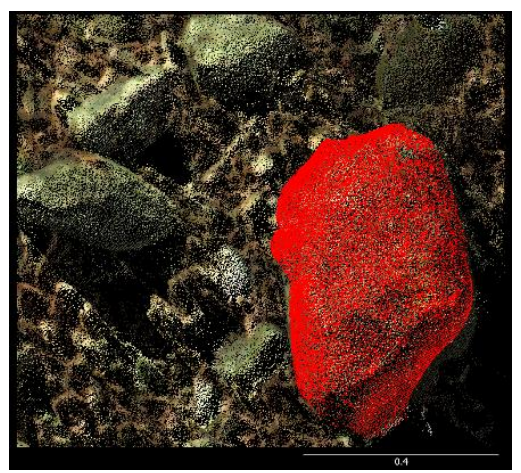

Figure 11 Position of point clouds after ICP registration aligned point cloud

\section{CONCLUSIONS}

In this study, a robust method for point clouds registration based on ICP algorithm has been proposed. The tests were conducted on terrestrial laser scanning point clouds representing river bed covered by rocks of different sizes. The proposed method involved key point detection and matching to obtain initial parameters of the transformation required by ICP algorithm. Due to the rounded, oblong shape of rocks, the key point detection was based on Gaussian curvature maxima detection.
The experiments showed, that the proposed algorithm allows for the registration of two point clouds even in the case of extremely adverse initial conditions such as:

- different acquisition campaign of the point clouds, which leads to object shape changes;

- oblong, rounded shape of the objects under registration;

- one of the point clouds represents wider scene than the other.

The investigations carried out on sample point clouds representing rocks enabled the adjustment of the parameters of the algorithm and showed that the Gaussian curvature can be used as a 3-dimentional key point detector for such objects. The proposed algorithm enabled to register point clouds with the mean distance between point clouds equal to $3 \mathrm{~mm}$ in both test and validation sets.

The proposed method is a vital part of the algorithm for monitoring the rock movement in the mountain river bed since it enables calculation of the rock movement parameters (translation and rotation) between two measurement epochs.

\section{REFERENCES}

Barnhart, T.B., Crosby, B.T., 2013. Comparing Two Methods of Surface Change Detection on an Evolving Thermokarst Using High-Temporal-Frequency Terrestrial Laser Scanning, Selawik River Alaska. Remote Sensing, 5, pp. 2813-2837.

Bergman, N., Laronne, J. B., Reid, I, 2007. Benefits of design modifications to the Birkbeck bedload sampler illustrated by flash-floods in an ephemeral gravel-bed channel. Earth Surface Processes and Landforms, 32(2), 317-328.

Bertin, S., Friedrich, H.,Delmas, P., Chan, E., Gimel'farb, G., 2015. Digital stereo photogrammetry for grain-scale monitoring of fluvial surfaces: Error evaluation and workflow optimization. ISPRS Journal of Photogrammetry and Remote Sensing, 101, pp. 193-208.

Ancey, C., Heyman, J., 2014. A microstructural approach to bed load transport: mean behaviour and fluctuations of particle transport rates. J. Fluid Mech. 744, 129-168.

Garcia, C., Laronne, J.B., Sala, M., 2000. Continuous monitoring of bedload flux in a mountain gravel-bed river. Geomorphology, $34,23-31$

Glira, P., Pfeifer, N., Briese, C., \& Ressl, C., 2015(4). A Correspondence Framework for ALS Strip Adjustments based on Variants of the ICP Algorithm. PhotogrammetrieFernerkundung Geoinformation, 275-289.

Jóźków, G., Borkowski, A., Kasprzak, M., 2016. Monitoring of fluvial transport in the mountain river bed using Terrestrial Laser Scanning. The International Archives of the Photogrammetry, Remote Sensing and Spatial Information Sciences, 41, 523-528.

Kuo, Ch-W., Brierley, G., Chang, Y-H. 2015. Monitoring channel responses to flood events of low to moderate magnitudes in a bedrock-dominated river using morphological budgeting by terrestrial laser scanning. Geomorphology, 235, 1-14.

Lotsari, E., Wang, Y., Kaartinen, H., Jaakkola, A., Kukko, A., Vaaja, M., Hyyppä, H., Hyyppä, J., Alho, P., 2015. Gravel transport by ice in a subarctic river from accurate laser scanning. Geomorphology. 246, 113-122. 
Olinde, L., Johnson, J. P. L., 2015. Using RFID and accelerometer embedded tracers to measure probabilities of bed load transport, step lengths, and rest times in a mountain stream. Water Resour. Res., 51(9), 7572-7589.

Picco, L., Mao, L., Cavalli, M., Buzzi, E., Rainato, R., Lenzi, M.A., 2013. Evaluating short-term morphological changes in a gravel-bed braided river using terrestrial laser scanner. Geomorphology, 201, 323-334.

Rusu, R. B., Cousins, S., 2011. 3D is here: Point Cloud Library (PCL). IEEE International Conference on Robotics and Automation (ICRA).

Zhong Y., 2009. Intrinsic shape signatures: A shape descriptor for 3D object recognition. IEEE 12th International Conference on Computer Vision Workshops, ICCV Workshops. 